\title{
Effect of Threatened Abortion on Fetal Growth and Premature Rupture of Membranes
}

Abdallah Khalil Ahmed ${ }^{1}$ MD, Samir Khamis Abd Elfattah Galal ${ }^{1}$ MD, Mohammed Hosny Ahmed Emara ${ }^{1 *}$ M.B.B.Ch

*Corresponding Author: Mohamed Hosny Emara drhgi2017@yahoo.com

Received for publication February 21, 2020; Accepted May 15, 2020; Published on line June 03, 2020.

Copyright 2020 The Authors published by Al-Azhar University, Faculty of Medicine, Cairo, Egypt. All rights reserved. This an openaccess article distributed under the legal terms, where it is permissible to download and share the work provided it is properly cited. The work cannot be changed in anyway or used commercially.

doi:

10.21608/aimj.2020.23981.1144

${ }^{1}$ Department of Obstetrics and Gynecology, Faculty of Medicine, Al-Azhar University, Cairo, Egypt

\begin{abstract}
Background: Threatened abortion is defined as bleeding without cervical dilatation before 20 weeks of gestation, and occurs in around $20 \%$ of recognized pregnancies. The aim of the study was to evaluate the effect of threatened abortion on fetal growth and premature rupture of membrane.

Patient and Methods: During a prospective case control study 200 selected pregnant women below 20 weeks gestation were assessed regarding presence of symptoms of threatened abortion. The diagnostic criteria for threatened abortion were based on documented fetal cardiac activity on ultrasound with history of vaginal bleeding in presence of a closed cervix and gestational age at 20 weeks or less.

Results: Our investigations showed that women with threatened miscarriage had a significantly increased risk of preterm labor (less 37 weeks of gestation) compared with the control group $(16 \% v s .2 \%, \mathrm{p}=$ $0.001)$. Women with threatened miscarriage had significantly small babies weighing less than 2500 gm compared with the control group, with a mean birth weight $(2335.1 \pm 644.9 v s .3118 .9 \pm 211.7, \mathrm{p}<0.0001)$. There was a significant difference regarding admission of the neonates to NICU ( $28 \%$ in the case group vs. $7 \%$ in the control group, $p=0.001$ ). There were no significant differences in the incidence of PROM, between the two groups.

Conclusion: The current study reports that patients with threatened abortion are at increased risk for adverse pregnancy outcomes as low birth weight, premature rupture of membrane.

Key words: Threatened abortion, Fetal growth, Premature rupture of membranes
\end{abstract}

Disclosure: The authors have no financial interest to declare in relation to the content of this article. The Article Processing Charge was paid for by the authors.

Authorship: All authors have a substantial contributions to the article

\section{INTRODUCTION}

The diagnosis of threatened abortion is frequently made in clinical practice because of taking a history of vaginal spotting and the finding of a closed cervix at subsequent vaginal examination. A definitive diagnosis of threatened abortion should be made following ultra sonographic examination, confirming the presence of fetal heart activity in an intrauterine pregnancy. ${ }^{1}$

Bleeding during pregnancy can cause maternal anxiety and emerging evidence suggests that it may be associated with poor fetal and maternal outcomes. ${ }^{2}$ Therefore, it is necessary to be diagnosed and managed to prevent maternal or fetal mortalities and morbidities. ${ }^{3}$

It is hypothesized that first-trimester bleeding may indicate an underlying placental dysfunction, which may manifest later in pregnancy causing adverse outcomes such as increased risk of pre-eclampsia, preterm delivery, preterm premature rupture of membranes (PPROM), placental abruption and intrauterine growth restriction (IUGR). ${ }^{4}$

While spontaneous rupture of membranes (ROM) is a normal component of labor and delivery, premature rupture of membranes (PROM) refers to rupture of the fetal membranes prior to the onset of labor irrespective of gestational age (can occur even at 42 weeks gestation). ${ }^{5}$

Premature rupture of membranes may occur at term or immediately preceding labour, or it may be an unexpected complication during the preterm period, when it is referred to as preterm premature rupture of membranes. ${ }^{6}$

Preterm Premature rupture of membranes (PROM) is a frequent obstetrical incident $(3 \%)$ which can result in maternal and fetal complications such as infection and prematurity. ${ }^{7}$

Pre-term, premature rupture of the membranes (PROM) is the most common cause of pre-term labour $(30-40 \%){ }^{8}$

The aim of the study is to evaluate the effect of threatened abortion on fetal growth and premature rupture of membrane. 


\section{PATIENTS AND METHODS}

The current prospective case-control study was conducted during a period of one year, starting from January 2019 till January 2020, which included two hundred pregnant women (100 cases and 100 controls), who were recruited in the study from the Outpatient Clinic of the Obstetrics and Gynecology Department, at Al Azhar university.

All patients of both cases and study groups were given $200 \mathrm{mg}$ progesterone supplementation twice daily in the form of rectal suppositories for a one week only.

Patients were divided in to 2 groups: Group 1 (Cases); one hundred women presented with symptoms of threatened abortion at or below 20 week of gestation, who had ultrasound examination afterwards. Group 2 (Controls); one hundred women who didn't have any symptoms of threatened abortion.

The diagnostic criteria for threatened abortion were based on documented fetal cardiac activity on ultrasound with history of vaginal bleeding in presence of a closed cervix and gestational age at 20 weeks or less.

Recruitment and procedures applied in the study: (i) Place of recruitment (Place of conduction of the study): subjects were recruited in the study from the outpatient Clinic of the Obstetrics and Gynecology Department, at Al-Hussein university hospital. (ii) Research Ethics Committee Approval and quality control; the study purpose and procedures were explained in details and in plain terms to each of the subjects before required to give an informed written consent to participate in the study. Quality control of screening, handling of data and verification of adherence to protocols were done on a regular basis by the trial coordinator. (iii) Subjects consent: all subjects, were informed about the details of the study, the risks and the benefits, and were all asked to give their consent before the start of the study. (iv) Procedures applied in the study: 1-History taking: including; personal history, menstrual history (to be sure for the last menstrual period and its gestational age), past history (for any previous pregnancy complications or medical disorders) and family history. 2-Examination: a careful examination and assessment was done with special attention to the inclusion and exclusion criteria among all couples, as follows; (a) General examination (focusing on the blood pressure to exclude pregnancy-induced hypertension [PIH], temperature and respiratory rate). (b) Body mass index, which was calculated according to the formula, $\mathrm{BMI}=\mathrm{Kg} / \mathrm{M}^{2}$. (c) Abdominal examination for fundal level and any sign of trauma. (d) Obstetric examination. 3-Investigations as follows; (a) Blood typing (ABO Grouping) and antibody testing ( $\mathrm{Rh}$ antibody, in cases of $\mathrm{Rh}$ negative). (b) Complete blood count (CBC). (c) Fasting blood glucose and 2-hours oral glucose tolerance test. (d) Urine analysis. (e) Thyroid, kidney and liver function tests. (f) Ultrasound assessment.
Ultrasound: Ultrasound probe was placed just cephalad to the symphysis pubis with the probe indicator oriented to the patient's right side. The probe was angled inferiorly so that the bladder is visualized, with the vaginal stripe and cervix posterior to the bladder. The probe was then tilted upward, keeping the indicator to the right, with appearance of the cervix and uterine fundus. The probe was continued to be tilted upward until the top of the uterus disappeared from view. After completing a transverse scan of the uterus, the probe was rotated clockwise so that the probe indicator was toward the patient's head, providing a longitudinal (or sagittal) view. In this view, the bladder was seen anteriorly and inferiorly, with the vaginal stripe posterior.

Sonographic parameters evaluated were: (a) Size of gestational sac and CRL if $<12$ weeks. (b) Fetal cardiac activity. (c) Subchorionic hematoma. (d) Fetal biometry: BPD, FL, AC if $>12$ weeks. (e) Placental site. (f) Amniotic fluid index.

Follow up: subjects in both groups were followed up every eight-week until delivery by US.

Outcome measures: (i)Primary outcome: occurrence of intrauterine growth restriction (IUGR) or premature rupture of membranes (PROM). (ii) Secondary outcome(s): occurrence of Placental abruption, preterm labor, neonatal sepsis and neonatal intensive care unit admission.

Statistical analysis: The data were coded, entered and processed on an IBM-PC compatible computer using statistical package for social sciences (SPSS) version $17, p$-value of $<0.05$ was considered significant. Unpaired Student's t-test was used to assess the statistical significance of the difference between two population means and medians in a study involving independent samples.

\section{RES ULTS}

In case group: patients who had spontaneous abortion $(n=17)$ and patients that could not be followed up $(n=25)$ were excluded and then we added 42 patients to the study with the same inclusion and exclusion criteria to complete the case group to 100 patients. (Table 1 )

In control group: patients who had spontaneous abortion $(n=1)$ and patients that could not be followed up $(n=15)$ were excluded from the study and then we added 16 patients with the same inclusion and exclusion criteria to complete the control group to100 patients. (Table 1)

Occurrence of intrauterine growth restriction (IUGR) or premature rupture of membranes (PROM), were the primary outcomes. (Table 1)

The demographic factors for the two groups are presented in Tables 1, 2 and 3. The mean maternal ages for the threatened miscarriage and the control groups were $28 \pm 6.5$ and $28.6 \pm 6.1$ years, ranged (18 to 40 ) years respectively. There was no 
significant difference within age distribution of the groups $(\mathrm{p}=0.5)$. The mean parity for the study and the control groups were 2.4 and 2.5 deliveries respectively $(\mathrm{p}=0.5)$. $($ Table 1$)$
The mean gestational age at presentation for the threatened miscarriage group was $15.4 \pm 2.4$ weeks compared to $16.3 \pm 2.2$ in the controlled group. (Table 1)

\begin{tabular}{|c|c|c|c|}
\hline Parameter (Variable) & $\begin{array}{c}\text { Group 1 } \\
(\text { Cases; } n=100)\end{array}$ & $\begin{array}{c}\text { Group 2 } \\
\text { (Controls; } n=100) \\
\end{array}$ & $\mathbf{P}$ \\
\hline Maternal age (years) & $28 \pm 6.5$ & $28.6 \pm 6.1$ & 0.5 \\
\hline Maternal BMI $^{(1)}\left(\mathrm{Kg} / \mathrm{M}^{2}\right)$ & $24 \pm 3.3$ & $25.3 \pm 2.8$ & $\mathbf{0 . 0 0 3}$ \\
\hline Gravidity & $3.6 \pm 1.2$ & $3.9 \pm 1.4$ & 0.1 \\
\hline Parity & $2.4 \pm 1.1$ & $2.5 \pm 1.1$ & 0.5 \\
\hline Gestational age $^{(2)}$ (weeks) & $15.4 \pm 2.4$ & $16.3 \pm 2.2$ & 0.006 \\
\hline Gestational age $^{(3)}$ (weeks) & $32.7 \pm 2.4$ & $33.8 \pm 3.3$ & 0.4 \\
\hline \multicolumn{4}{|l|}{ Fetal biometry $(\mathrm{mm})$} \\
\hline BPD & $32.5 \pm 7.5$ & $36.5 \pm 7.3$ & 0.0002 \\
\hline $\mathrm{AC}$ & $100.7 \pm 22.5$ & $117.6 \pm 25.4$ & $<0.0001$ \\
\hline FL & $19.9 \pm 6.6$ & $25 \pm 6.3$ & $<0.0001$ \\
\hline \multicolumn{4}{|l|}{ Mode of delivery } \\
\hline Cesarean section & 61 & 29 & \multirow{2}{*}{0.1} \\
\hline Vaginal delivery & 39 & 71 & \\
\hline
\end{tabular}

Table (1): Comparison between both groups as regards the characteristics

(1) BMI; Body Mass Index. (2) Gestational age at time of presentation (examination).

(3) Gestational age at time of rupture of membrane.

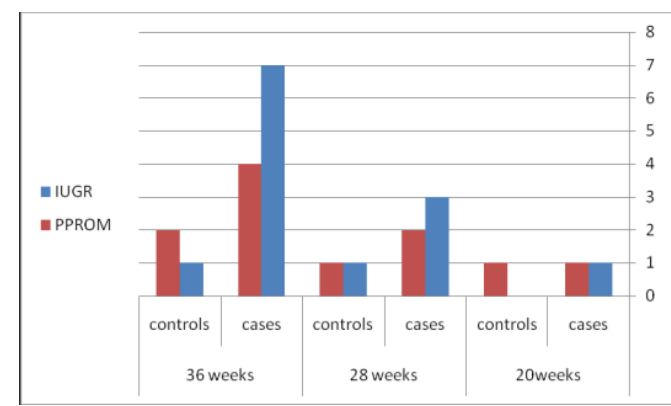

Fig (1): Comparison between both groups as regards the characteristics

(1) BMI; Body Mass Index. (2) Gestational age at time of presentation (examination).

(3) Gestational age at time of rupture of membrane.

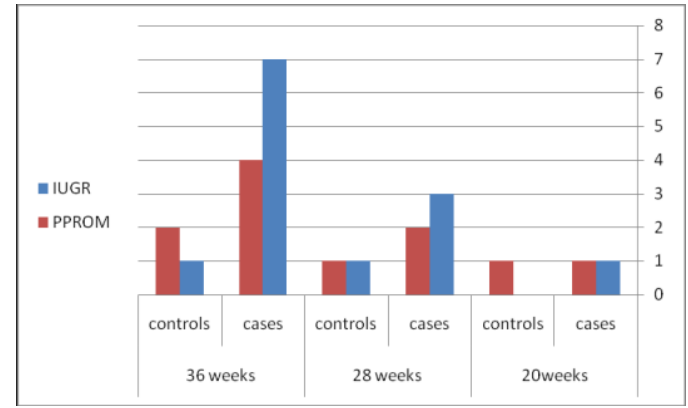

Fig (2): Comparison between both groups follow up according to primary outcomes at 20 weeks, 28 weeks $\& 36$ weeks

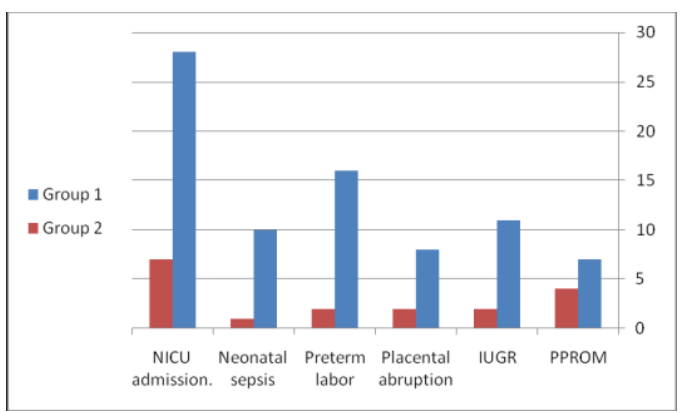

Fig (3): Comparison between both groups as regards the primary and secondary outcome measures

(1)PPROM; Preterm Premature Rupture of Membranes.

(2)IUGR; Intra-Uterine Growth Restriction.

(3)NICU; neonatal intensive care unit

(4) NICU; neonatal intensive care unit.

The overall adverse pregnancy outcomes were significantly higher in women with threatened miscarriage than the control group.

Our investigations showed that women with threatened miscarriage had a significantly increased risk of preterm labor (less 37 weeks of gestation) compared with the control group ( $16 \%$ vs. $2 \%, \mathrm{p}=$ 0.001). Women with threatened miscarriage had significantly small babies weighing less than 2500 gm compared with the control group, with a mean birth weight $(2335.1 \pm 644.9$ vs. $3118.9 \pm$ 
211.7, $\mathrm{p}<0.0001)$. There was a significant difference regarding admission of the neonates to NICU ( $28 \%$ in the case group vs. $7 \%$ in the control group, $\mathrm{p}=0.001)$. There were no significant differences in the incidence of PROM, between the two groups

\section{DISCUSSION}

Our study showed no significant difference regarding the maternal age as the mean maternal age in the case group was $(28 \pm 6.5)$ years vs. $(28.6 \pm 6.1)$ in the control group with $\mathrm{p}$ value 0.5 ; but Davari-Tanha et al. ${ }^{9}$ showed that the mean maternal age was $(27.13 \pm$ $4.76)$ in case group vs. $(26.5 \pm 4.52)$ in control group with $\mathrm{p}$ value 0.014 .

Our study showed a significant difference regarding the maternal body mass index as the mean maternal body mass index was $(24 \pm 3.3)$ in the case group compared to $(25.3 \pm 2.8)$ in the control group with $\mathrm{p}$ value 0.003 . Other studies did not refer to the maternal body mass index.

Our study showed no significant difference regarding the gravidity as the mean gravidity in case group was $(3.6 \pm 1.2)$ vs. $(3.9 \pm 1.4)$ in the control group with $p$ value 0.1 ; but Davari-Tanha et al. ${ }^{9}$, reported a significant difference regarding the gravidity between the case and control group with $p$ value $<0.001$, On contrary to that Dadkhah et al. ${ }^{10}$, found no relation between threatened abortion and gravidity.

Our study showed also no significant difference regarding the parity as the mean parity in the case group was $(2.4 \pm 1.1)$ vs. $(2.5 \pm 1.1)$ in the control group with $\mathrm{p}$ value 0.5 . Dadkhah et al. ${ }^{10}$, found no relation between threatened abortion and parity.

Our study showed a significant difference regarding the gestational age (weeks) at time of presentation / examination as the mean gestational age in the case group at presentation was $(15.4 \pm 2.4)$ while it was $(16,3 \pm 2.2)$ in the control group with $p$ value 0.006 but Davari-Tanha et al. ${ }^{9}$ reported that the median onset of bleeding was 9th and 10th week of gestation in the high risk group and the low risk group respectively.

Our study showed, there was a relative increase in the rate of PPROM in the case group vs. in the control group $(7 \%, 4 \%)$ respectively with $(\mathrm{P}$ value $0.5)$.

Davari-Tanha et al. ${ }^{9}$ and Saraswat et al. ${ }^{11}$ reported a significant statistical difference regarding PPROM with ( $\mathrm{p}$ value $0.001,0.01$ ) respectively.

Although the cause is unclear, it is hypothesized that disruption of the chorionic amniotic plane by adjacent hemorrhage that may make the membrane more susceptible to rupture Batzofin et al. ${ }^{12}$ alternatively, the prolonged presence of blood may act as a nidus for intrauterine infection.

Our study showed a significant statistical difference in the incidence of IUGR in the case group than in the control group (11\% vs. $2 \%)$ respectively with (p value 0.02 ) and we also noted that infants of patients with threatened abortion had nearly $300 \mathrm{gm}$ difference in birth weight when compared with the control group with ( $\mathrm{p}$ value $<0.0001)$.

Davari-Tanha et al. ${ }^{9}$ reported an increased risk for IUGR in the case group ( $\mathrm{p}$ value 0.0888 ).

Haddow et al. 13 reported an increased risk for low birth weight $<2500 \mathrm{gm}$ in pregnancies complicated by threatened abortion.

Our study showed a relative increase in the incidence of hypertensive disorders as the incidence was $6 \%$ in the case group compared to $2 \%$ in the control group ( $\mathrm{p}$ value 0.2 ).

This result was similar to the study done by DavariTanha et al. ${ }^{9}$ as the incidence was $4.6 \%$ in case group vs. $9.8 \%$ in the control group with (p value 0.121). In 2009, Hasan et al. ${ }^{14}$ reported that pregnancy induced hypertension was significantly more common in the case group compared to the control group (6\% vs. $4.7 \%$, respectively (p value < 0.05).

Our data showed that there was a relative increase in the incidence of placenta previa in the case group than in the control group (4\% vs. $0 \%$ respectively, $\mathrm{p}$ value 0.1 ) but Davari-Tanha et al. ${ }^{9}$ showed there was no increase in the incidence of placenta previa ( $p$ value 1 )

Das et al. ${ }^{15}$ reported an increased risk for low lying placenta among patients with threatened abortion but reported no difference in placental location compared with control group by 36 weeks of gestation.

Also, our study reported a relative increase in the incidence of cesarean section in case group than in control group (39\% vs. $29 \%$ respectively, p value $0.1)$.

Davari-Tanha et al. ${ }^{9}$ reported also a relative increase in the rate of cesarean section in case group than in control group ( $42.8 \%$ vs. $52 \%$, p value 0.453 ).

Finally, our study showed a significant difference regarding the neonatal admission to NICU as the incidence was $28 \%$ in the case group vs. $7 \%$ in the control group with $\mathrm{p}$ value 0.001 , and this was similar to the study done by Saraswat et al. ${ }^{11}$ who reported a significant difference regarding the NICU admission between both groups with $\mathrm{p}$ value 0.009 . 
The two studies mentioned above Davari-Tanha et al. ${ }^{9}$ and Saraswat et al. ${ }^{11}$ was different from our study as that our study was done on patients of threatened abortion at or below 20 weeks of gestation but the others did it on patients of threatened abortion in the first trimester only.

In the present study, significantly suboptimal outcome was greater for preterm birth (before 37 weeks of gestation) and low birth weight. Preterm labor and premature rupture of membranes may be considered one phenomenon, because both conditions are intermingling. The low birth weight and lower Apgar score observed in the study cases is a logic sequence of preterm birth and possibly a lower level of antenatal care given. It seems that all of the above adverse pregnancy outcomes are consequences of PROM.

There is evidence that later pregnancy complications such as preterm labor and premature rupture of the membranes may be due to impaired placentation and more recently reactive oxygen species as a result of early pregnancy insult ${ }^{16}$.

\section{CONCLUSION}

The current study reports that patients with threatened abortion are at increased risk for adverse pregnancy outcomes as low birth weight, premature rupture of membrane (PROM), so the physicians should be aware of the adverse outcomes that may be associated with threatened abortion and should be alert for signs of these complications.

\section{REFERENCES}

1. Park IY, Park CH, Lee G, et al. Prognosis of threatened abortion by embryonic/fetal heart beat rate. Ultrasound Med Biol., 2006; 32(5):264.

2. Wijesiriwardana A, Bhattachary a S, Shetty A, et al. Obstetric outcome in women with threatened miscarriage in the first trimester. Obstet Gynecol., 2006;107:557-62.

3. Mulik V, Bethel J and Bhal K. A retrospective population-based study of primigravid women on the potential effect of threatened miscarriage on obstetric outcome. J Obstet Gynaecol., 2004; 24:249-53.

4. Weiss JL, Malone FD, Vidaver J, et al. Threatened abortion: a risk factor for poor pregnancy outcome, a population based screening study. Am J Obstet Gynecol., 2004; 190:745-50.

5. Caughey $\mathrm{AB}$, Robinson $\mathrm{JN}$ and Norwitz ER. Contemporary diagnosis and management of preterm premature rupture of membranes. Rev Obstet Gynecol., 2008; 1(1):11-22.

6. El Messidi A and Cameron A. Diagnosis of premature rupture of membranes inspiration from the past and insights for the Future. J Obstet Gynecolcan., 2010; 32 (6): 561-69.
7. Mercer BM. Preterm premature rupture of the membranes. Obstet Gynecol., 2003; ;101:17893.

8. Dinsmoor MJ, Bachman R, Haney EI, et al. Outcomes after expectant management of extremely preterm premature. Am J Obstet Gynecol., 2010; 81: 336-411.

9. Davari-Tanha F, Shariat M, Kaveh M, et al. Threatened abortion a risk factor for poor pregnancy outcome. Acta Media Iranica, 2008; 46(4):314-20.

10. Dadkhah F, Kashanian $M$ and Eliasi GA. Acomparison between the pregnancy outcpme in women with or without threatened abortion. Early Human Dev., 2010; 86:193-96.

11. Saraswat L, Bhattacharya S, Maheshwari A, et al. Maternal perinatal outcome in women with threatened miscarriage in the first trimester:a systemic review. BJOG., 2010; 117:245-57.

12. Batzofin JH, Fielding WL and Friedman EA. Pregnancy outcome of threatened abortion with demonstrable fetal cardiac activity: a cohort study. Obstet Gynecol., 1995; 63(4):515-18.

13. Haddow JE, Knight GJ, Kloza EM, et al. Alphafetoprotein, vaginal bleeding and pregnancy risk.Br J Obstet Gynaecol., 1996; 93(6):589-93.

14. Hasan R, Baird DD, Herring AH, et al. Association between first-trimester vaginal bleeding and miscarriage. Obstet Gynecol., 2009; 114(4):860-67.

15. Das AG, Gopalan S and Dhaliwal LK. Fetal growth and perinatal outcome of pregnancies continuing after threatened abortion. Aust NZ J Obstet Gynaecol., 1996; 36(2):135-39.

16. Plessinger MA, Woods JR and Miller RK. Pretreatment of human amnion-chorion with vitamins $\mathrm{C}$ and $\mathrm{E}$ prevents hypochlorous acidinduced damage. Am J Obstet Gynecol., 2014; 183(4):979-85. 